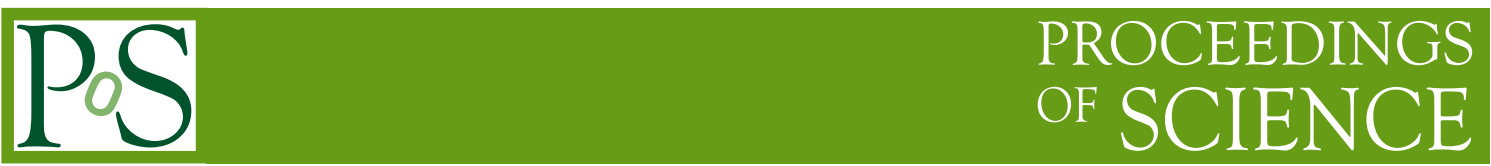

\title{
Current Status of Transverse Spin at STAR
}

\author{
Andrew Gordon* for the STAR Collaboration \\ Brookhaven National Laboratory \\ E-mail: agordonebnl.gov
}

\begin{abstract}
The origins of the proton spin remain an area of active investigation. The Relativistic Heavy Ion Collider (RHIC) at Brookhaven National Lab uniquely provides polarized proton data at high center of mass energies. STAR has previously reported large transverse single spin asymmetries for inclusive neutral pion production in the forward direction at $\sqrt{s}=200 \mathrm{GeV}$, measured with a modular Pb-glass calorimeter called the "Forward Pion Detector" (FPD). Beginning in 2008 (Run 8), STAR's forward calorimetry was significantly enhanced with the commissioning of the "Forward Meson Spectrometer" (FMS), which has full azimuthal coverage in the pseudo-rapidity range $2.5<\eta<4.2$. This detector has extended asymmetry measurements to higher $P_{T}$, and its large areal coverage enables measurements of higher mass resonances and "jet-like" correlations. STAR also provides excellent tracking and full azimuthal electromagnetic calorimeter coverage at mid-rapidity, which facilitates analyses of spin-dependent jet structure. Here we review recent transverse spin measurements with the STAR detector and also present an update on some existing analyses.
\end{abstract}

XVIII International Workshop on Deep-Inelastic Scattering and Related Subjects, DIS 2010 April 19-23, 2010

Firenze, Italy

\footnotetext{
* Speaker.
} 


\section{Introduction}

STAR has previously reported precision measurements [1] of the analyzing power $\left(A_{N}\right)$ of inclusive neutral pions in the forward region. These measurements used data taken in RHIC runs 3, 5, and 6 with the Forward Pion Detector (FPD), a modular Pb-glass array that can be moved horizontally in the plane transverse to the beamline. These data were taken at $\sqrt{s}=200 \mathrm{GeV}$, where inclusive $\pi^{0}$ cross sections are consistent with expectations from pQCD [2]. The measurements showed that the variation of $A_{N}$ with Feynman-x $\left(X_{F}=2 P_{L} / \sqrt{s}\right)$ was qualitatively consistent with expectations from the Sivers effect [3], while the $P_{T}$ dependence was not. The extent to which the Sivers and Collins [ 4 , 5] effects contribute to these measurements has yet to be determined. FPD measurements of inclusive $\eta$ production have also been reported, with the data indicating a strong growth of $A_{N}$ with increasing $X_{F}$ [6].

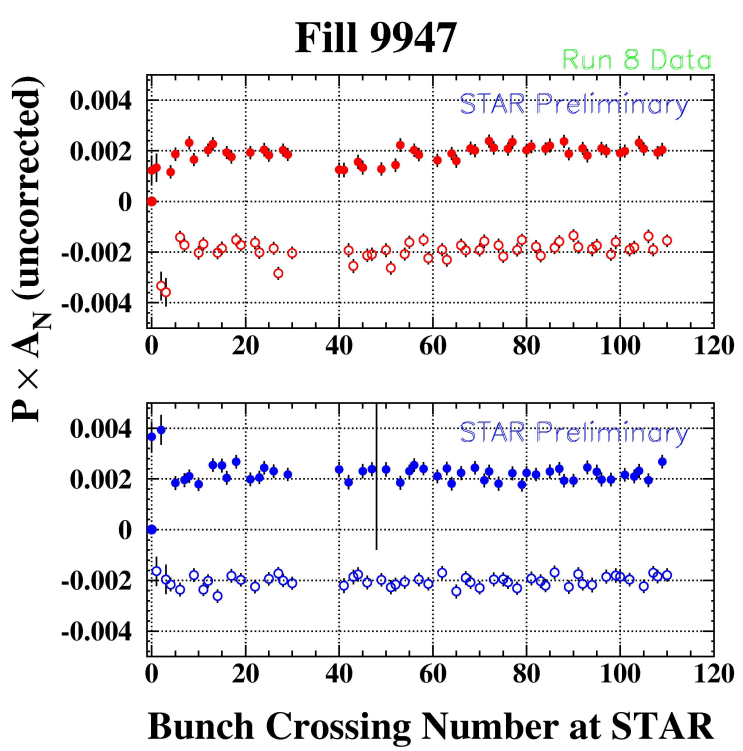

Figure 1: Observed transverse single spin asymmetry (uncorrected) for individual bunches as seen by the STAR BBCs, for one RHIC fill in Run 8. Top plot is for the beam headed eastward at STAR, and the bottom is for the beam headed westward. The solid circles are for bunches that were prepared spin up and the open circles for spin down. The observed asymmetry was calculated with a procedure similar to [7], and the mean over all the bunches are set to have 0 asymmetry for each beam.

STAR has also observed forward analyzing powers with its "Beam-BeamCounters" (BBC), at rapidities from $3.9<$ $\eta<5$.0. Single transverse spin asymmetries in the detection of charged hadrons with these detectors can be used as a "local polarimeter" [7]. Figure 11 shows the bunch-by-bunch relative transverse polarization as seen at STAR, for one RHIC fill in 2008 (Run 8). All fills have been examined and provide confirmation that the bunches have relatively uniform absolute polarization, and that the expected bunch-by-bunch spin pattern is also the spin pattern observed at STAR. The local polarimeter also allows studies of possible variations in the bunch-by-bunch polarization within a fill.

The Sivers effect identifies the origin of the observed spin asymmetries with orbital motion of the quarks inside the polarized proton. By contrast, in the Collins effect the polarization of the struck quark is correlated to the polarization of the proton, and the fragmentation of the polarized quark leads to asymmetries within

the resulting jet.

STAR commissioned a new detector in 2008 for Run 8, the Forward Meson Spectrometer (FMS) [8]. The FMS is a nearly hermetic array of $1264 \mathrm{~Pb}$-glass blocks situated $\sim 700 \mathrm{~cm}$ downstream of the interaction point and spanning an area $200 \times 200 \mathrm{~cm}^{2}$ perpendicular to the beam pipe. It covers the full azimuth in the range $2.5<\eta<4.0$ and provides a many-fold increase in the areal 
coverage of the forward region at STAR [B]. This larger detector enhances the ability to extend the previously published inclusive $\pi^{0}$ data [1], and also allows the measurements of higher mass resonances, including the $\omega[9]$ and $J / \psi[10]$.

The inclusive $\pi^{0} A_{N}$ was measured using the FMS in Run 8 , and the $X_{F}$ dependence of this data has provided an important confirmation of the previously published data [11, 12]. In addition, the FMS has extended STAR's $P_{T}$ reach in the forward direction. The Run 8 data agree with the previous data and also indicate that the analyzing power in the forward region remains large to at least $P_{T}>4 \mathrm{GeV}$. Moreover, the FMS has allowed studies of two- $\pi^{0}$ events. Studies of the azimuthal difference $\Delta \phi\left(\pi^{0}, \pi^{0}\right)$ of all observed $\pi^{0} \pi^{0}$ pairs in the FMS show two peaks, one at $\Delta \phi=0$ and one at $\Delta \phi=\pi$, indicating that a hard-scatter event underlies these data.

\section{On-going Analyses}

The Collins effect is consistent with string fragmentation models in which a quark/anti-quark pair is produced with relative orbital angular momentum at the point of string breaking [14 15]. This leads to an asymmetry within a jet in the the production of the spin 0 pions, and would lead to the opposite asymmetry for spin 1 particles [16]. The decay of the spin- $1,782 \mathrm{MeV} \omega$ is accessible with the FMS through the $\omega \rightarrow \pi^{0} \gamma$ decay channel (BR=8.9\% [13]), and observation of a negative $A_{N}$ might provide strong experimental confirmation of these theoretical models.

The preliminary analysis of $\omega \rightarrow \pi^{0} \gamma$

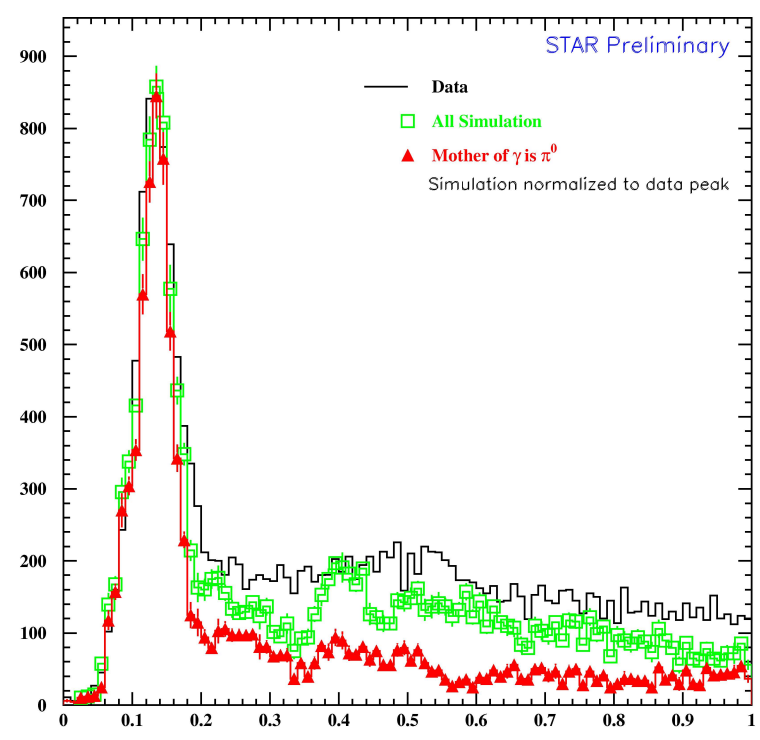

Figure 2: Distribution of $M\left(\gamma, 4^{\text {th }}\right.$ cluster) (see text) for events with four or more clusters, for data (black lines), Pythia(6.2.22)+GEANT simulation (green squares), and the subset of the simulation for which the mother of the $\omega$-associated photon is really a $\pi^{0}$ (red triangles). The green points are scaled to the data at the peak position. decays in the FMS for RHIC Run 8 has been described elsewhere [9]. Two photon clusters derive from the $\pi^{0}$ decay and one directly from the $\omega$. For each set of three electromagnetic (EM) clusters in the FMS there are three possible pairs that can be associated with the $\pi^{0}$ decay. The pair whose mass is closest to $0.135 \mathrm{GeV} / \mathrm{c}^{2}$ is associated with the $\pi^{0}$ decay, while the third is associated with the $\gamma$. Simulations show that this procedure tags the photons correctly upwards of $99 \%$ of the time. Minimum energy and $P_{T}$ cuts are placed on the clusters to help reduce backgrounds from non-photonic particles.

A signal of $\approx 10$ statistical standard deviations is evident in the data, although with a signal to background ratio of roughly 1:5 [9]. This high background level limits the ability to detect a negative analyzing power. If we assume that the beam polarization is $50 \%$ and the background has $A_{N}=0$, then the Run 8 data would be able to detect a negative $A_{N}$ with $>2$ statistical standard deviations for $A_{N}(\omega)<-0.3$. 
Pythia(6.2.22)+GEANT simulations [9] predict that the largest background source of fake $\omega \rightarrow \pi^{0} \gamma$ decays are from events where the $\gamma$ derives from $\pi^{0}$ decay and not from an $\omega$. There are also significant contributions from $\eta \rightarrow \gamma \gamma$, as well as from events where the cluster that was assumed to be the $\gamma$ actually derived from a particle that is not a photon. The inclusive $\pi^{0}$ and $\eta$ data sets show large, positive $A_{N}$ and also show a strong energy dependence, and the $\omega A_{N}$ is thus sensitive to the precise composition of the background.

There is evidence that the Pythia+GEANT simulation significantly underestimates the nonphotonic background contribution to the three-EM cluster sample. To see this we look at events that have a set of three clusters that pass the $\omega$ cuts [2], but that also have at least one extra EM cluster in the event. We then consider the cluster that we associated with the $\gamma$ for the $\omega \rightarrow \pi^{0} \gamma$ decay, and we calculate the mass of that cluster with one of the extra clusters. We refer to this mass as $M\left(\gamma, 4^{\text {th }}\right.$ cluster $)$. We calculate this separately for all extra clusters in the event.

Figure 2 shows the distribution of $M\left(\gamma, 4^{\text {th }}\right.$ cluster $)$ for data and the Pythia+GEANT simulation. The peak at $0.135 \mathrm{GeV} / \mathrm{c}^{2}$ is from events where the set of three clusters is actually drawn from the four photons of a $\pi^{0} \pi^{0}$ event. The simulation shows significantly fewer events in the tail of the distribution relative to the data, when the simulation is scaled so that the peak height matches the data. This indicates that the data may contain significantly more non-photonic EM clusters than the simulation.

STAR is also sensitive to the Collins asymmetry through jet reconstruction. Spin dependence in the fragmentation of a transversely polarized quark in a transversely polarized proton (the Collins effect) can be manifested in azimuthal dependence of $\pi^{+/-}$production within the jet around the jet axis, relative to the direction of the transversely polarized proton spin [17]. The measured asymmetry depends on $\phi_{\pi}$ and $\phi_{S}$ where $\phi_{\pi}$ is the azimuth of the pion above the reaction plane, and $\phi_{S}$ is the azimuth of the transversely polarized proton spin relative to the reaction plane. Here the reaction plane is spanned by a vector along the transversely polarized beam direction and a vector along the jet axis.

The asymmetry can depend on $z=P_{\pi} / P_{j e t}$ and also on $j_{t}$, the component of the pion momentum transverse to the jet axis. The asymmetry is calculated separately for $\pi^{+}$and $\pi^{-}$. Since a single spin asymmetry is being measured, one of the RHIC beams is averaged over, while the spin state of the other beam is used for $\phi_{S}$. Only jets in the forward hemisphere are considered, where forward is defined relative to the proton beam whose spin state is being considered. Calculations are done separately for averaging over the spin states of each of the two beams.

For this analysis, mid-rapidity jets are used. STAR's Time Projection Chamber (TPC) [18] provides charged particle tracking with good particle identification for momenta up to $\approx 15 \mathrm{GeV}$. In addition the "barrel" (BEMC, $-1<\eta<1$ ) [19] and "endcap" (EEMC, $1<\eta<2$ ) [20] calorimeters provide EM energy measurements and are used for jet reconstruction and also for triggering.

The expected statistical power of transverse data taken in 2006 (Run 6) is expected to be better than around \pm 0.01 . By comparison a simulation based on expectations for transversity and Collins fragmentation from global fits to HERMES, COMPASS, and BELLE data [21] give a rough expectation for the asymmetry of $|A| \approx 0.03$. Systematic uncertainties currently under study include effects from trigger biases, contamination of the $\pi^{+/-}$sample from other particles, tracking efficiencies, accuracy of jet and pion kinematic measurements, beam polarization, and relative luminosities for the different beam polarization states. 


\section{Conclusion and Outlook}

Large single spin asymmetries have been observed at STAR in the forward region, for both inclusive $\pi^{0}$ and $\eta$ signals. Run 8 measurements have extended the $P_{T}$ range for inclusive $\pi^{0}$ at high $X_{F}$ and also confirmed the previous measurements. In addition, an observation of a negative analyzing power for $\omega \rightarrow \pi^{0} \gamma$ in the FMS would provide an exciting test of a theoretical understanding of the Collins effect. STAR is also analyzing mid-rapidity jets to provide measurements of the Collins effect.

Future upgrades to the forward region might allow jet triggering, which would allow direct measurements of the Sivers effect in the forward region. In addition, STAR has reported measurements of $J / \psi$ production in the forward region [10], raising the question of the degree to which Drell-Yan production of $e^{+} e^{-}$pairs might also be accessible in the future in the mass range above $\approx 3 \mathrm{GeV} / \mathrm{c}^{2}$.

\section{References}

[1] B.I. Abelev et al,Phys. Rev. Lett. 100, 232003 (2008).

[2] J. Adams et al, Phys. Rev. Lett. 97, 152302 (2006).

[3] D. Sivers, Phys. Rev. D 41, 93 (1990).

[4] J. C. Collins, Nucl. Phys. B 396, 161 (1993).

[5] J. Collins, S. Heppelmann, G. Ladinsky, Nucl. Phys. B 420, 565 (1994).

[6] S. Heppelmann, for the STAR collaboration, arXiv:0905.2840 (DIS 2008 proceedings).

[7] J. Kiryluk, for the STAR collaboration, arXiv:hep-ex/0501072 (2005).

[8] N. Poljak, for the STAR collaboration arXiv:0901.2828 (SPIN 2008 proceedings).

[9] A. Gordon, for the STAR collaboration arXiv:0906.2332 (Moriond 2009 proceedings).

[10] C. Perkins, for the STAR collaboration arXiv:0907.4396 (Quark Matter 2009 proceedings).

[11] J. L. Drachenberg, arXiv:0901.2763, AIP Conf. Proc 1149517 (2009).

[12] A. Ogawa, for the STAR collaboration, AIP Conf.Proc 1182561 (2009) (CIPANP 2009 proceedings).

[13] W.-M. Yao et al. (Particle Data Group), J. Phys. G 33, 1 (2006).

[14] X. Artru and J. Czyżewski, H. Yabuki, hep-ph/9508239vl (1995).

[15] X. Artru and J. Czyżewski, hep-ph/9805463vl (1998).

[16] X. Artru, arXiv:1001.1061 (2010).

[17] F. Yuan, Phys. Rev. Lett. 100, 032003 (2008).

[18] M. Anderson et al, Nucl. Instrum. Methods A 499, 659 (2003).

[19] M. Beddo et al, Nucl. Instrum. Methods A 499, 725 (2003).

[20] C. E. Allgower et al, Nucl. Instrum. Methods A 499, 740 (2003).

[21] M. Anselmino et al., Phys. Rev. D 75, 054032 (2007). 